\title{
Property study of a new silica nanoparticle delivery system of hydrophobic phthalocyanine using spectroscopic method
}

\author{
Rui Lin ${ }^{\text {a }}$, Lin Zhou ${ }^{\text {a }}$, Yun Lin ${ }^{\mathrm{a}}$, Ao Wang ${ }^{\mathrm{a}}$, Jia Hong Zhou ${ }^{\mathrm{a}, \mathrm{b}}$ and Shao Hua Wei ${ }^{\mathrm{a}, *}$ \\ a Jiangsu Key Laboratory Biofunctional Materials, College of Chemistry and Materials Science, \\ Nanjing Normal University, Nanjing, China \\ ${ }^{\mathrm{b}}$ Analysis Testing Center, Nanjing Normal University, Nanjing, China
}

\begin{abstract}
Tetra-tert-butyl zinc(II) phthalocyanine has attracted great attention as an effective photosensitizer in the field of photodynamic therapy. However, tetra-tert-butyl zinc(II) phthalocyanine is hydrophobic and trends to aggregate to form the inactive congeries in aqueous solution, which dramatically reduce its photodynamic activity. To solve this problem, tetratert-butyl zinc(II) phthalocyanine was encapsulated into silica nanoparticles using microemulsion method and its properties were studied using spectroscopic methods. After embedded inside the silica nanoparticles, the water-solubility, stability and monomer percent of phthalocyanine in water were greatly improved comparing with the free phthalocyanines, and therefore, its photo-induced singlet oxygen generation efficacy of drug-doped silica nanoparticles was improved. The results indicated that encapsulation hydrophobic phthalocyanine into silica nanoparticles was an effective way to solve their problems and promote their clinical application.
\end{abstract}

Keywords: Phthalocyanine, nanocomposites, photodynamic therapy, ceramics

\section{Introduction}

Photodynamic therapy (PDT) is a promising treatment for several types of cancers, such as esophageal cancer, liver cancer and so on. This treatment involves the administration of photosensitizers (PSs), and then irradiates with light of a specific wavelength to induce cell death. Singlet oxygen $\left({ }^{1} \mathrm{O}_{2}\right)$ and other cytototoxic reactive oxygen species (ROSs) are formed in the overall reaction [10-12].

One of the PSs most widely used in PDT is phthalocyanines (Pcs) because of their strong absorption in the phototherapeutic window, high singlet oxygen generation efficiency, and high photodynamic activities for inactivation of different tumour cell lines $[1,2,8,13]$. However, it was found that the preparation of injectable formula for Pcs was highly hampered by the poor water solubility of these compounds. In addition, Pcs will aggregate in aqueous solution, which can interfere with their PDT efficacy and decrease the fluorescence of the Pcs [16].

To solve this problem, approaches such as structural modifications with hydrophilic or amphipathic groups have been attempted $[9,15]$. In vitro studies indicated that such hydrophilicity or amphipathic derivatives show superior photo-induced anti-cancer activity to the original Pcs $[3,18]$. However, the

\footnotetext{
${ }^{*}$ Corresponding author: Shao Hua Wei, Jiangsu Key Laboratory Biofunctional Materials, College of Chemistry and Materials Science, Nanjing Normal University, Nanjing 210046, China. Tel.: +86 25 85891761; Fax: +86 25 85891761; E-mail: shwei@njnu.edu.cn.
} 


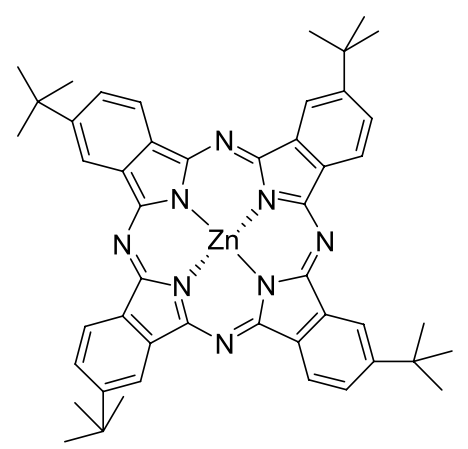

Fig. 1. Chemical structure of tetra-tert-butyl zinc(II) phthalocyanine.

structure modification approach involves complicated synthetic and purification steps, which are not general and always possible. As an alternative way, preparation of nano-scale drug delivery system can also solve above problems of phthalocyanines. The hydrophobic core of the nanocarrier provides a naturally compatible environment for loading hydrophobic Pcs molecules, while the hydrophilic shells stabilize the nanocarriers for increased water solubility and prolong blood circulation. Another advantage of incorporating Pcs-based PS agents into micelles includes the ability to prevent aggregation to avoid the loss of their PDT efficacy and fluorescence [5,17,21]. Recently, silica-based nanoparticles have been receiving quickly increased research attentions as Ps delivery system because of their superior biocompatibility and the controllable size, shape, porosity and mono-dispersibility properties [7].

Here, tetra-tert-butyl zinc(II) phthalocyanine (TBPc) (Fig. 1) as the representative of lipophilic phthalocyanine, was encapsulated into silica nanoparticle (TBPcSN) by the microemulsion way. In addition, the morphology and size, optimal encapsulation amount, singlet oxygen production properties of TBPcSN were studied using spectroscopic methods.

\section{Materials and methods}

\subsection{Materials}

TBPc was obtained from Sigma-Aldrich. Surfactant triethoxyvinylsilane (TEVS; 97\%) and 3-aminopropyltriethoxysilane (APTES; 99\%) were purchased from ACROS. Cetyltrimethylammonium bromide (CTAB; 99\%), cosurfactant 1-butanol (99\%), chloroform $\left(\mathrm{CHCl}_{3}\right)$ were purchased from Sinopharm chemical reagent.

\subsection{TBPCSN preparation}

TBPcSN was prepared by microemulsion method [4,19] with some modification. Briefly, to $20 \mathrm{ml}$ of doubly distilled water, $0.36 \mathrm{~g}$ of CTAB and $800 \mu \mathrm{l}$ of 1-butanol was added. Then, different volumes of TBPc solution in $\mathrm{CHCl}_{3}\left(10^{-2} \mathrm{M}\right)$ was added by magnetic stirring. Next, $200 \mu \mathrm{l}$ of TEVS was added and the resulting solution was stirred until it became clear (about $1 \mathrm{~h}$ ). After that, $20 \mu \mathrm{l}$ of APTES was added and the system was stirred for about $20 \mathrm{~h}$ in dark. The entire reaction was carried out at room temperature. After the formation of the nanoparticles, surfactant CTAB and cosurfactant 1-butanol were removed by dialyzing the solution against water with a 12-14 kDa molecular weight cutoff cellulose membrane for $48 \mathrm{~h}$. As the control, free TBPc was dissolved in water using chloroform $\left(\mathrm{CHCl}_{3}\right)$ as latent solvent. 


\subsection{Characterization}

Transmission electron microscopy (TEM) image was obtained using a JEM 2010(HR) electron microscope. All absorption spectra were recorded on a VARIAN CARY 5000 UV-Vis spectrophotometer. Fluorescence spectra were obtained by a PERKIN-ELMER LS-50B fluorospectrophotometer [20].

Photoinduced singlet oxygen generation was determined by photobleaching of the chemical probe 9,10-anthracendipropionic acid (ADPA). ADPA can be photo-bleached by singlet oxygen to its corresponding endoperoxide and the process was monitored by spectrophotometrically by recording the decrease in optical density at $379 \mathrm{~nm}$ ( $\lambda_{\max }$ of ADPA). In a typical experiment, an aqueous solution of ADPA $(100 \mathrm{ml}, 5.5 \mathrm{mmol})$ was mixed with TBPcSN $(3 \mathrm{ml}, 0.1 \mu \mathrm{mol})$. The control experiment used ADPA mixed with aqueous solution of TBPc, which has been prepared by adding small amounts of concentrated $\mathrm{CHCl}_{3}$ solutions of TBPc to doubly distilled water. These solutions were irradiated with a $500 \mathrm{~W}$ high-voltage mercury lamp. The optical densities at $378 \mathrm{~nm}$ (characteristic absorption peak of ADPA) were recorded every $30 \mathrm{~s}$ using VARIAN Cary $5000 \mathrm{UV}$-Vis spectrophotometer.

\section{Results and discussion}

The TEM images (Fig. 2) clearly showed that the TBPcSN were spherical, uniform and monodispersed with sizes of about $25-30 \mathrm{~nm}$. Such ultralow size (less than $50 \mathrm{~nm}$ ) can help them evade capture by the RES [14].

What's more, in order to comparing the stability, we measured the absorption spectrum of two aqueous solutions of TBPc and TBPcSN as a function of dosage time (Fig. 3). It clearly showed that the stability of TBPcSN was increased compared to free TBPc, which indicated that TBPcSNs has excellent stability in the aqueous solution due to their ultrasmall size.

The UV-Vis absorption spectra (Fig. 4) showed that TBPc aqueous solution had two low peaks, located at 684 and $635 \mathrm{~nm}$, ascribed to the monomer and the congeries of TBPc, separately. However, TBPcSN had a clear Q-band at $679 \mathrm{~nm}$, the characteristic peak of monomer, suggested little aggregation of TBPc, which was quite similar as TBPc in oily solution [6]. This phenomenon indicated that the TBPc

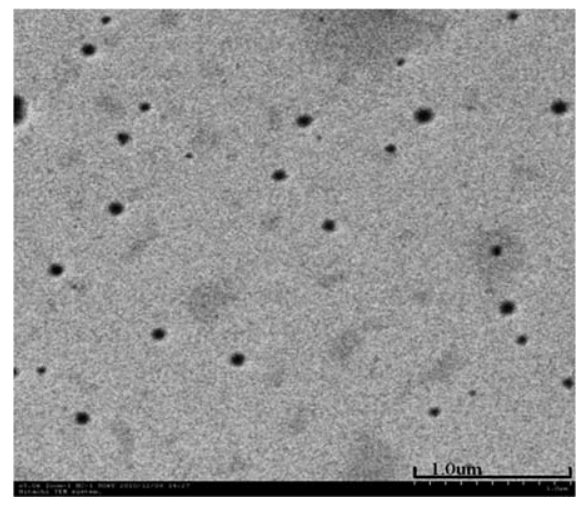

Fig. 2. TEM images of TBPcSN.

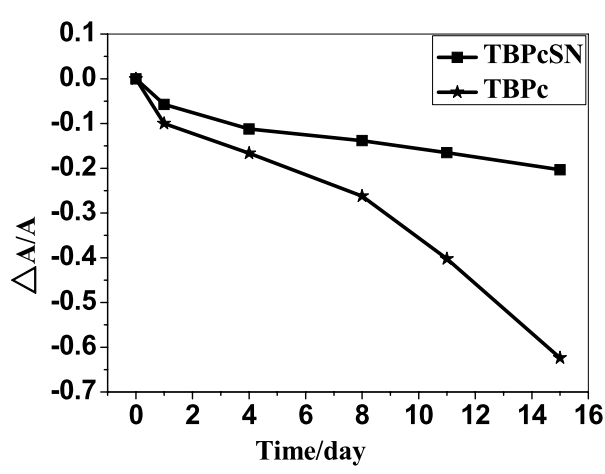

Fig. 3. The stability comparing of TBPcSN and TBPc in aqueous solution by detecting the absorption changes at $679 \mathrm{~nm}$ for $\mathrm{TBPcSN}$ and $684 \mathrm{~nm}$ for TBPc as a function of dosage time. 


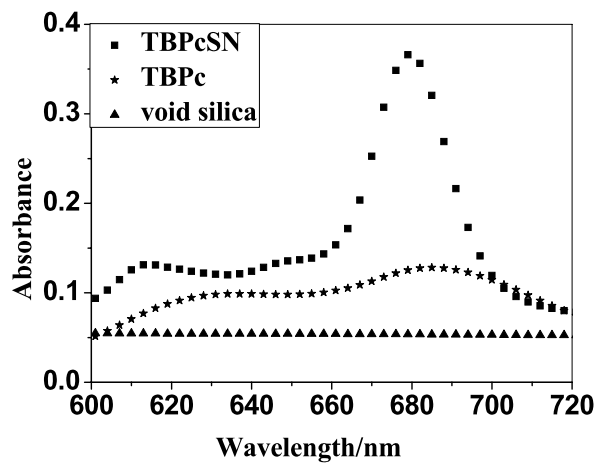

Fig. 4. UV-Vis absorption spectra TBPcSN, TBPc in aqueous solution and void silica nanoparticles.

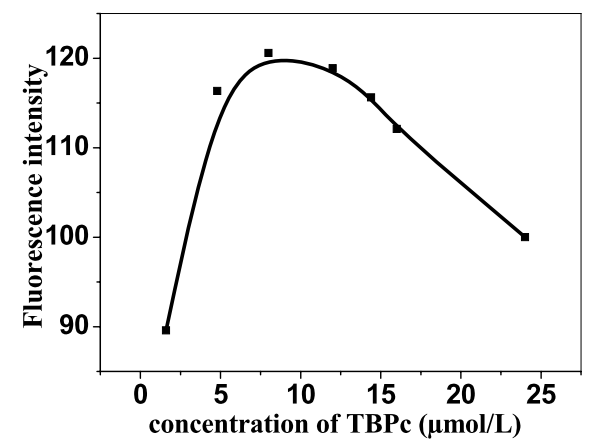

Fig. 6. The fluorescence intensity of different concentrations of TBPcSN.

molecules were successfully encapsulated inside the silica nanoparticles and the silica matrix provided a relative hydrophobic environment to prevent TBPc aggregation. In addition, the percent of the monomer form of TBPc, which is the efficient form to generate reactive oxygen species, was greatly improved after encapsulation inside the silica nanoparticles.

In addition, TBPcSN had a strong fluorescence peak locating at about $688 \mathrm{~nm}$ (Fig. 5) in aqueous solution, further indicated that TBPc were successfully embedded inside the nanocarrier and such relative hydrophobic environment avoided the congeries formation of TBPc. In contrast, the same concentration of TBPc and the void silica nanoparticles aqueous solution exhibited practically no emission.

Furthermore, it was found that the fluorescence intensity of TBPcSN was dependent on the concentration of embedded TBPc. When the embedding concentration of TBPc was $8 \mu \mathrm{M}$, the fluorescence intensity reached a maximum (Fig. 6). At a higher TBPc concentration, the fluorescence of TBPcSN dropped, which could be related to a fluorescence quenching effect due to the self-aggregation of TBPc molecules. Therefore, the optimized concentration of TBPc in the TBPcSN was $8 \mu \mathrm{M}$.

In order to get more evidence to prove that TBPc was encapsulated inside the silica nanoparticles, $\mathrm{CHCl}_{3}$ was used to extract the photosensitizer from TBPcSN or TBPc aqueous solution (Fig. 7). By monitoring the absorption of the $\mathrm{CHCl}_{3}$ phase over time, the TBPc concentration in $\mathrm{CHCl}_{3}$ within $25 \mathrm{~min}$ 
in TBPc aqueous solution system was increased. In contrast, almost no TBPc absorption was detected in $\mathrm{CHCl}_{3}$ phase of the TBPcSN system, which further verified that TBPc was successfully embedded inside the silica nanoparticles.

Singlet oxygen is believed to play a key role in the efficacy of PDT. The formation of ${ }^{1} \mathrm{O}_{2}$ was measured by the bleaching of ADPA dye, which was monitored by following the net loss of ADPA absorption at $378 \mathrm{~nm}$ over time. The absorption intensity of ADPA continuously decreased as the irradiation time increasing, the generation of singlet oxygen by TBPcSN and TBPc was confirmed (Fig. 8a and b). By
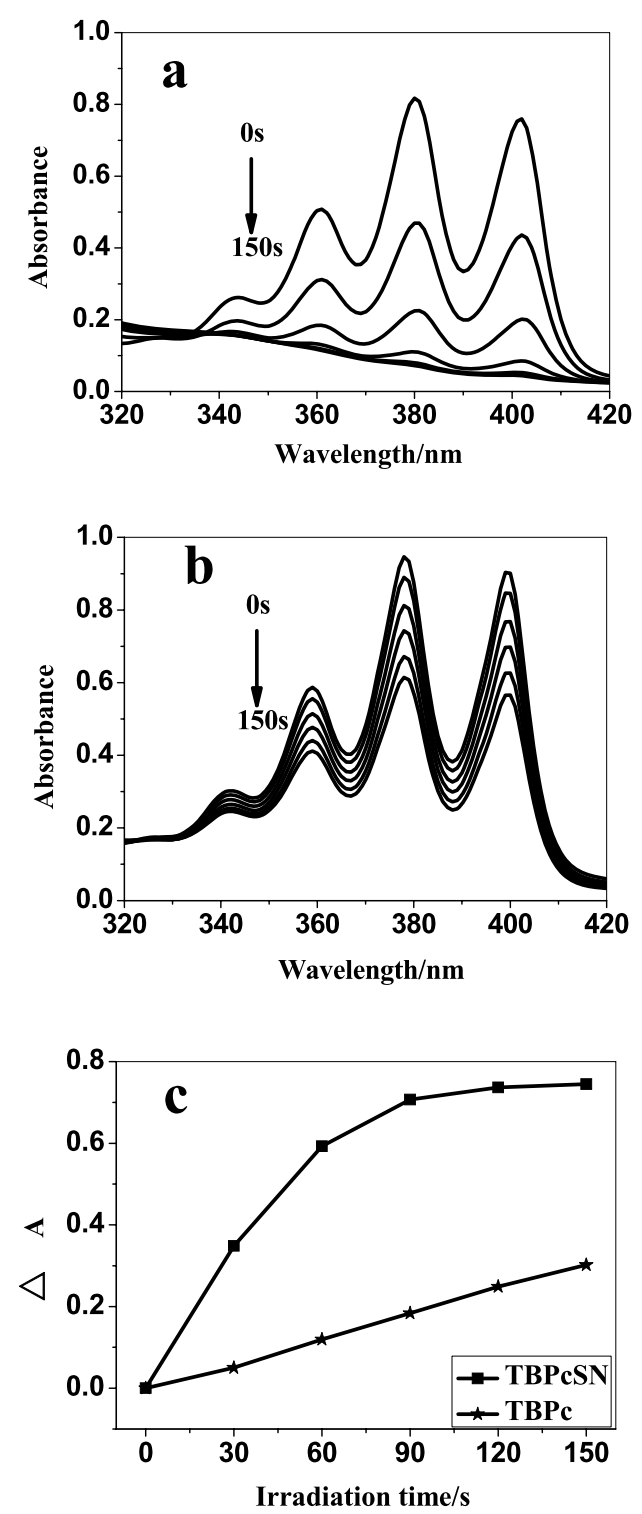

Fig. 8. Absorbance spectra of ADPA water solution containing (a) TBPcSN and (b) TBPc as a function of irradiation times $(0-150 \mathrm{~s})$, (c) the photo-induced absorbance intensity decay $(\Delta A)$ curves of ADPA at $378 \mathrm{~nm}$ to monitor the singlet oxygen generated by TBPcSN and TBPc. 
comparing with TBPc, the singlet oxygen delivery of TBPc was enhanced after been encapsulated inside the silica nanocarrier (Fig. 8c) because the nanocarrier protected and retained the long-lived singlet state of TBPc.

\section{Conclusions}

In summary, the results demonstrate the successful embedding of TBPc in silica nanoparticles prepared through the reversed microemulsion method and its properties were studied using spectroscopic methods. After encapsulated inside the silica nanoparticles, the water solubility, stability, monomer percent and ability of singlet oxygen generation were greatly improved. Above results indicated that encapsulation hydrophobic phthalocyanine into silica nanoparticles was an effective way to solve their problem and promoted their clinical application.

\section{Acknowledgements}

This work was supported by the National Natural Science Foundation of China (20973093 and 20673958) and the Priority Academic Program Development of Jiangsu Higher Education Institutions (PAPD).

\section{References}

[1] H. Ali and J.E. Van Lier, Metal complexes as photo- and radiosensitizers, Chem. Rev. 99 (1999), 2379-2450.

[2] C.M. Allen, W.M. Sharman and J.E. Van Lier, Current status of phthalocyanines in the photodynamic therapy of cancer, J. Porphyr. Phthalocya 5 (2001), 161-169.

[3] G. Andras, B. Peter, B. Istvan, C. Viktor, K. Mikos, S. Klara et al., Triple state properties of tetrasubstituted zinc phthalocyanine derivatives, J. Mol. Struct. 704 (2004), 11-15.

[4] R.P. Bagwe, C. Yang, L.R. Hilliard and W. Tan, Optimization of dyedoped silica nanoparticles prepared using a reverse microemulsion method, Langmuir 20 (2004), 8336-8342.

[5] P.A. Barbugli, M.P. Siqueira-Moura, E.M. Espreafico and A.C. Tedesco, In vitro photoxicity of liposomes and nanocapsules containing chloroaluminum phthalocyanine on human melanoma cell line, J. Nanosci. Nanotechnol. 10 (2010), 569-573.

[6] M.T.M. Choi, P.P.S. Li and D.K.P. Ng, A direct comparison of the aggregation behavior of phthalocyanines and 2,3naphthalocyanines, Tetrahedron 56 (2000), 3881-3887.

[7] P. Couleaud and V. Morosini, Silica-based nanoparticles for photodynamic therapy applications, Nanoscale 2 (2010), 1083-1095.

[8] M.R. Detty, S.L. Gibson and S.J. Wagner, Current clinical and preclinical photosensitizers for use in photodynamic therapy, J. Med. Chem. 47 (2004), 3897-3915.

[9] J.L. Huang, N.S. Chen, J.D. Huang, E.S. Liu, J.P. Xue and S.L. Yang, Metal phthalocyanine as photosensitizer for photodynamic therapy (PDT) preparation, Sci. China Ser. B 44 (2001), 113-122.

[10] J.G. Levy and M. Obochi, New applications in photodynamic therapy introduction, J. Photochem. Photobiol. 64 (1996), 737-739.

[11] F.X. Liu, X.P. Zhou, Z.L. Chen, P. Huang, X.Q. Wang and Y. Zhou, Preparation of purpurin-18 loaded magnetic nanocarriers in cottonseed oil for photodynamic therapy, Mater. Lett. 62 (2008), 2844-2847.

[12] L.M. Moreira, J.P. Lyon, S.M.S. Tursi, I. Trajano, M.P. Felipe et al., Azure dyes as new photosensitizer prototypes to application in photodynamic therapy against Candida spp., Spectrosc. Int. J. 24 (2010), 621-628.

[13] E.S. Nyman and P.H. Hynninen, Research advances in the use of tetrapyrrolic photosensitizers for photodynamic therapy, J. Photochem. Photobiol. B 73 (2004), 1-28.

[14] I. Roy, T.Y. Ohulchanskyy, H.E. Pudavar, E.J. Bergey, A.R. Oseroff, J. Morgan et al., Ceramic-based nanoparticles entrapping water-insoluble photosensitizer anticancer drugs: a novel drug-carrier system for photodynamic therapy, J. Am. Chem. Soc. 125 (2003), 7860-7865. 
[15] K. Sakamoto, T. Kato, E. Ohno-Okumura, M. Watanabe and M.J. Cook, Synthesis of novel cationic amphiphilic phthalocyanine derivatives for generation photosensitizer using photodynamic therapy of cancer, Dyes Pigm. 64 (2005), 63-71.

[16] J.P. Taquet, C. Frochot, V. Manneville and B.H. Muriel, Phthalocyanine covalently bound to biomolecules for a targeted photodynamic therapy, Curr. Med. Chem. 14 (2007), 1673-1687.

[17] S. Wang, R. Gao, F. Zhou and M. Selke, Nanomaterials and singlet oxygen photosensitizers: potential applications in photodynamic therapy, J. Mater. Chem. 14 (2004), 487-493.

[18] S.H. Wei, J.H. Zhou, D.Y. Huang, X.S. Wang, B.W. Zhang and J. Shen, Synthesis and Type I/Type II photosensitizing properties of a novel amphiphilic zinc phthalocyanine, Dyes Pigm. 71 (2006), 61-67.

[19] X.L. Xing, X.X. He, J.F. Peng, K.M. Wang and W.H. Tan, Uptake of silica-coated nanoparticles by hela cells, J. Nanosci. Nanotechnol. 5 (2005), 1688-1693.

[20] J.H. Zhou, X.H. Wu, C. Yang, X.T. Gu, L. Zhou et al., Spectroscopic studies on the interaction of hypocrellin A with myoglobin, Spectrosc. Int. J. 21 (2007), 235-243.

[21] L. Zhou, C. Dong, S.H. Wei, Y.Y. Feng, J.H. Zhou and J.H. Liu, Water-soluble soft nano-colloid for drug delivery, Mater. Lett. 63 (2009), 1683-1685. 


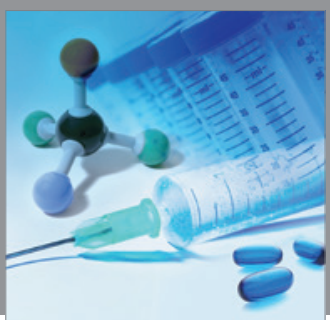

International Journal of

Medicinal Chemistry

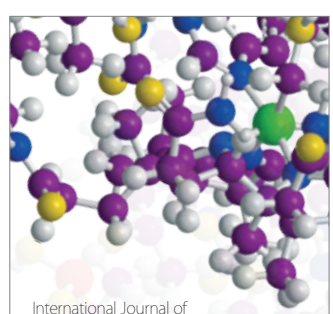

Carbohydrate Chemistry

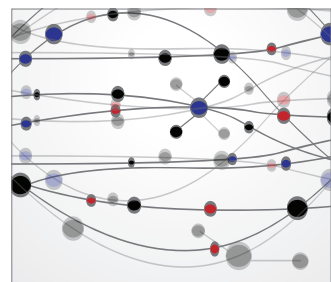

The Scientific World Journal
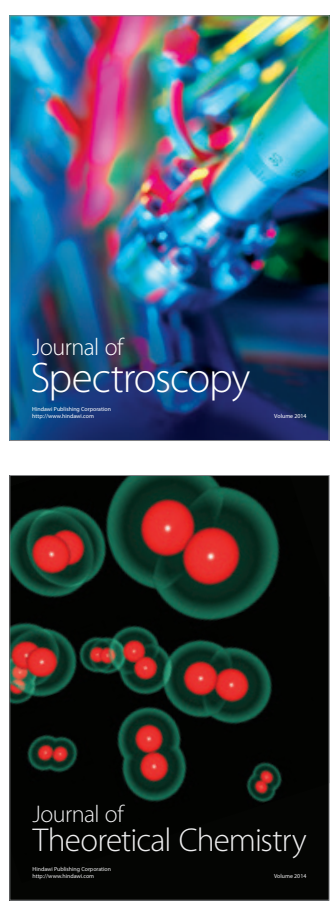
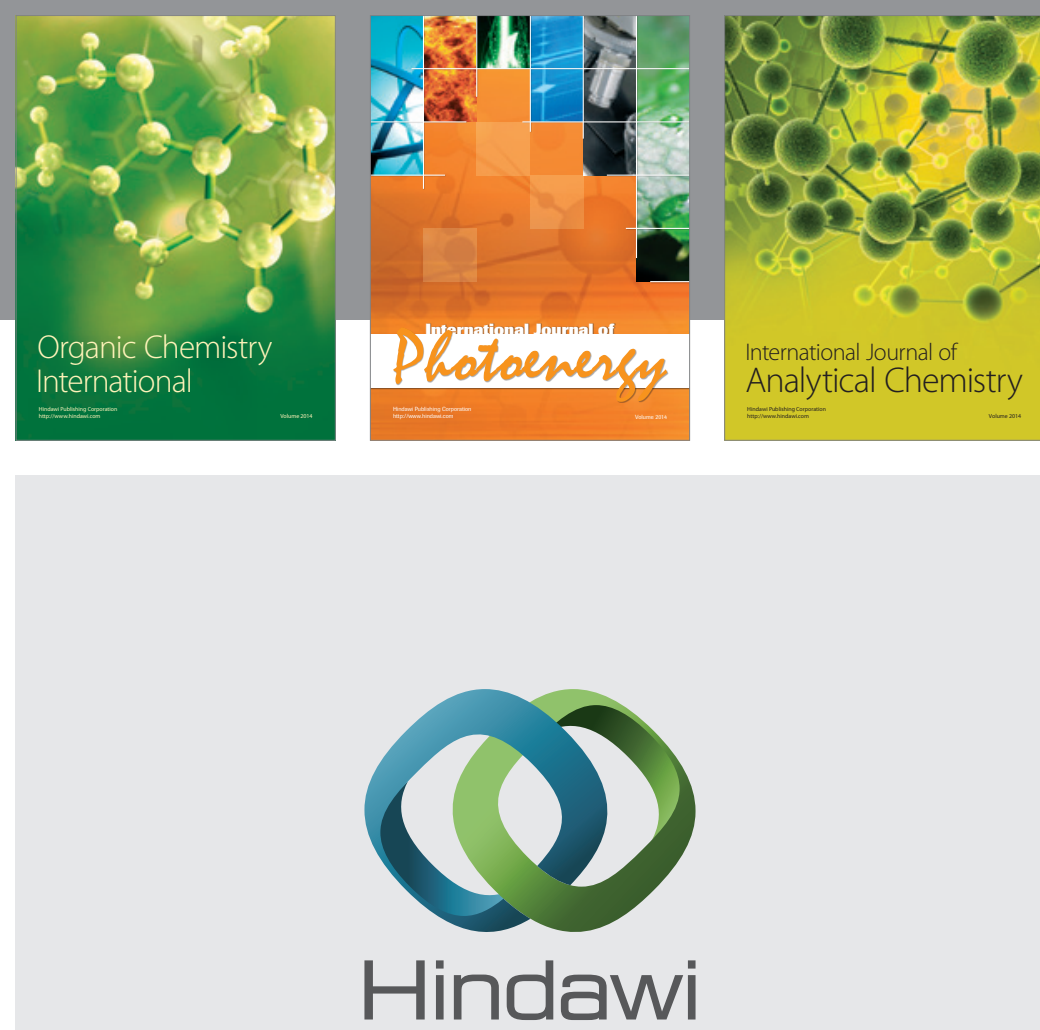

Submit your manuscripts at

http://www.hindawi.com
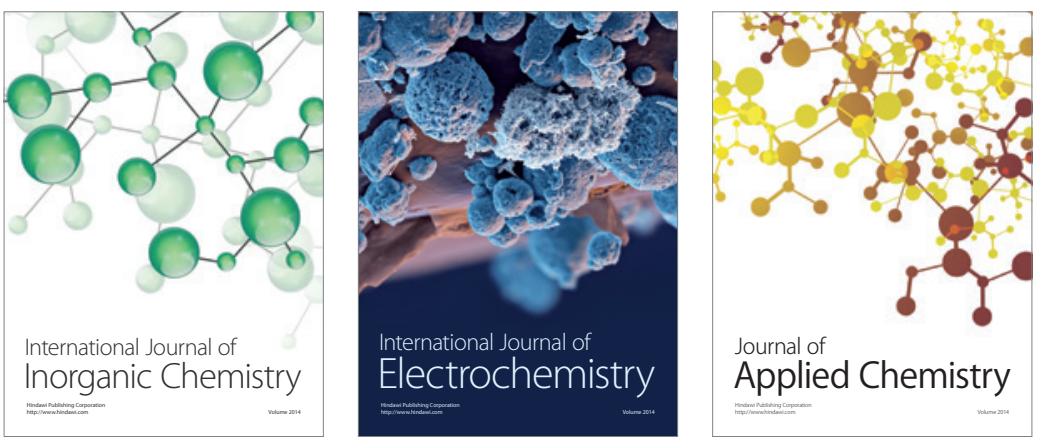

Journal of

Applied Chemistry
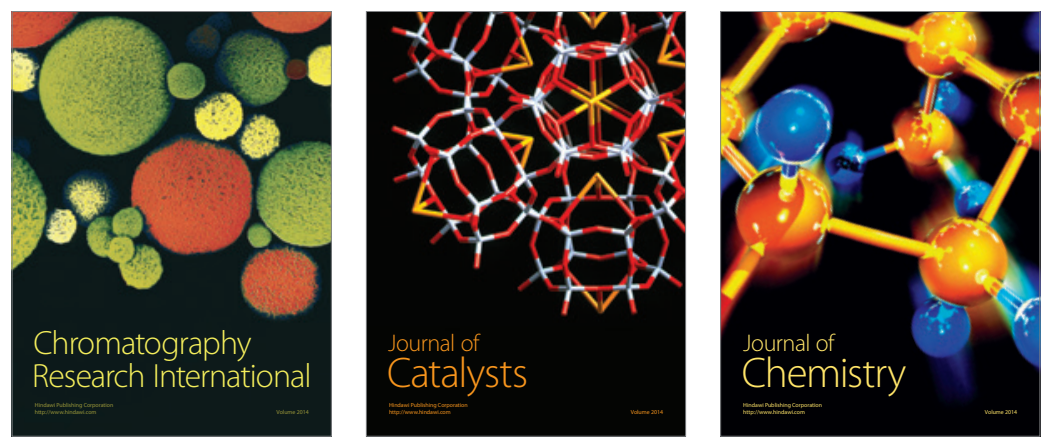
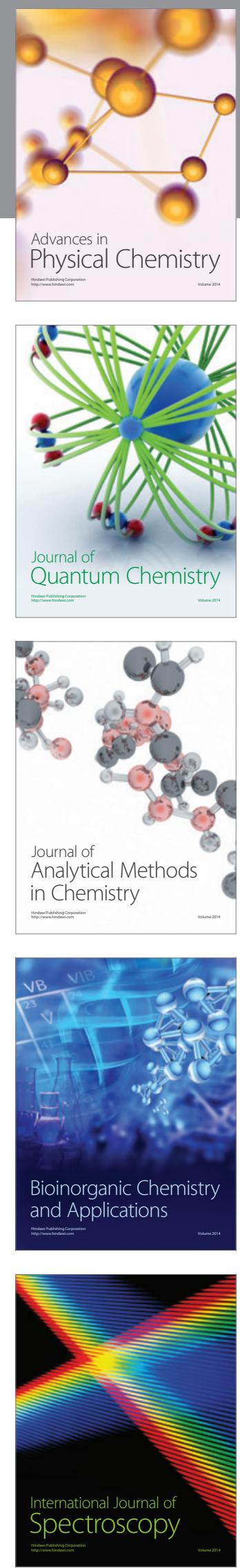